\title{
¿FUE PEDRO ABELARDO \\ UN EXTERNISTA MORAL?
}

\author{
WAS PETER ABELARD \\ A MORAL EXTERNALIST?
}

\author{
GONZALO MATA GARCÍA* \\ Universidad de Santiago de Compostela
}

RESUMEN: En este artículo se pretende analizar, de modo sistemático, la cuestión acerca del posible externismo de Pedro Abelardo. Se pone de relieve la filosofía moral del filósofo del siglo XII desde la perspectiva actual de la metaética, así como la cuestión de las razones para actuar y la motivación moral que podemos extrapolar a la actualidad.

PALABRAS ClaVE: externismo, moral, metaética, motivación moral

ABSTRACT: In this paper we hope to carry out a systematic analysis of the Abelardo's externalism. The main focus here is the moral philosophy of P. Abelard from a presentday metaethic perspective, and the reasons for acting and the moral motivation that we can extrapolate today.

KEYWORDS: externalism, moral, metaethic, moral motivation.

\section{Introducción}

El objetivo de este artículo es evaluar los posicionamientos de Pedro Abelardo en lo que respecta a dos problemas particularmente relevantes para la filosofía moral actual, como son el de la naturaleza de las propiedades morales y la relación entre motivación moral y normatividad. Las razones para tal investigación

\footnotetext{
*E-mail: gonzalo.mata@rai.usc.es
} 
son dobles. Por una parte, esta puede ayudarnos a comprender mejor, desde la nueva perspectiva que nos proporcionan los desarrollos actuales en metaética y psicología moral, la concepción abelardiana de la moral. Por otra parte, de manera inversa, el examen de las posiciones de Abelardo podrá servirnos para ver sobre una luz diferente el problema de la naturaleza de nuestras razones para actuar y la motivación moral.

El modo en el que nos interesa abordar aquí la cuestión no es historiográfico, sino sistemático. Buscamos considerar la posición abelardiana fundamentalmente en la medida en que su análisis nos sirva para evaluar su contribución al progreso que quepa hacer en el desarrollo de la filosofía moral actual.

Antes de pasar al apartado segundo se expondrán los conceptos más significativos para poder desarrollar con cierta facilidad las ideas posteriores, que se ordenarán en cuatro secciones más. Así, bajo el número dos se procederá a definir los términos relativos a las razones para actuar y la motivación moral con especial hincapié en la relevancia del uso de estas categorías para la filosofía moral. Esto irá seguido de un apartado en donde se lleve a cabo una introducción a la filosofía moral abelardiana focalizándolo en el rol que se asigna a la intención por parte de Pedro Abelardo, así como la ontología moral que asume. Tras ello ya se estará en disposición de ahondar en el tema principal del presente artículo, de modo que el punto de mira se fijará en el posicionamiento del filósofo medieval con respecto a lo que hoy consideramos una posición externista o internista de la motivación moral. Finalmente se expondrán las conclusiones finales que den respuesta al interrogante planteado; estas serán particularmente relevantes en lo que respecta al concepto de intención, y, de la mano de este, a los límites de la relevancia de la responsabilidad moral en el examen de la naturaleza de la moral.

En el corazón de la metaética radica la cuestión del estatuto ontológico de las razones mediante las que la ética normativa busca informar a la moral sobre cómo debemos actuar. Asimismo, la metaética y la psicología moral han de informarnos de la relación entre tales razones (o nuestra asunción de tales razones) y el hecho de que actuemos efectivamente conforme a ellas. Podríamos partir de la base de que la metaética se ocupa del análisis del sentido y justificación de los conceptos éticos, de modo que se trata de un discurso que a su vez se encarga de otro discurso, el discurso moral. A ello añadiremos que el modo en el que en la 
actualidad se formula esta cuestión viene a coincidir con la enunciación del problema general del internismo y el externismo. En principio, si dejamos de lado caracterizaciones más detalladas, podría caracterizarse al internismo indicando que este es la posición conforme a la cual poseemos una cierta razón para actuar, o, si se prefiere, un deber, o una razón normativa si y sólo si estamos motivados para obrar conforme a lo que tal razón (o deber) prescribe. En contrapartida, el externismo defendería, como cabe esperar, la posición contraria. Conforme a este, tenemos ciertas razones para actuar que son independientes de nuestra motivación para obrar conforme a ellas. Así, se negaría que haya un vínculo necesario entre la posesión de razones para actuar y la motivación para actuar conforme a ellas.

Estas definiciones podrían considerarse muy ambiguas; demasiado, de hecho. El motivo radica en que hay al menos dos tesis diferentes implicadas en las definiciones arriba dadas. Conforme a esto, vamos a encontrarnos con dos sentidos muy distintos que pueden tener los términos internismo y externismo en metaética y psicología moral. Por este motivo, ambos requerirán un análisis por separado. Una de las tesis concierne a la ontología de nuestras razones últimas para actuar. La otra, que estará vinculada con esta pero que será de carácter distinto, tiene que ver con nuestra concepción de la conexión entre motivación y normatividad.

\section{Razones para actuar y motivación moral}

Comencemos, pues, con las ideas que tienen que ver con la naturaleza de las prescripciones que hemos de seguir. En este sentido cabrá distinguir las siguientes posiciones. Así, el internismo de las razones para actuar sería la aceptación de la proposición (IR) ${ }^{1}$ :

(IR) Nuestras razones para actuar son exclusivamente de carácter interno. No tenemos razones externas para actuar. Esto es, una presunta razón para

\footnotetext{
${ }^{1}$ El internismo de las razones ha sido defendido, por ejemplo, en Williams, B., «Internal and External Reasons», en Moral Luck: Philosophical Papers 1973-1980, Cambridge University Press, Cambridge, 1981, 101-13; o Persson, I., The Retreat of Reason: A Dilemma in the Philosophy of Life, Oxford University Press, Oxford, 2005.
} 
actuar sólo puede serlo si el agente susceptible de poseerla la reconoce como tal, o reconoce otras razones para actuar con las cuales la razón en cuestión tiene un vínculo causal.

Definido de esta manera el internismo, el externismo de las razones para actuar no sería más que la posición contraria a este, esto es, la proposición (ER):

(ER) Nuestras razones para actuar no son exclusivamente de carácter interno. Tenemos razones externas para actuar. ${ }^{2}$

Conforme a esta posición, es posible tener obligaciones aun sin que lo sepamos. En otras palabras, este punto de vista implica que el realismo moral es cierto: existen determinadas propiedades morales (sean estas susceptibles de ser descritas en términos naturalistas o no) externas a su reconocimiento por nuestra parte, que nos proporcionan razones para obrar de un modo u otro. De hecho, podría ser incluso el caso que explícitamente rechazásemos tener un determinado deber y, sin embargo, lo tuviésemos.

En otras palabras, según el primer planteamiento existe una conexión «interna» entre el juicio evaluativo de que algo debería hacerse y su motivación para realizarlo. El internismo sostiene que el sujeto (la persona) es, o puede ser, consciente de la creencia justificada que le lleva a realizar la acción. Por el contrario, el externismo considera que no existe relación interna esencial entre las creencias morales y los motivos (para llevarlas a cabo), no existiendo de este modo razón por la cual la creencia de que algo es incorrecto lleve a desear no llevarlo a cabo. Según esto, el internalismo sostendría que la persona depende de sus propiedades intrínsecas, mientras que el externismo apoya la idea de que para tener algunos estados mentales intencionales (como las creencias) es necesario relacionarse con el entorno de modo correcto.

Una vez que hemos reconocido el problema de nuestras razones para actuar, nos hemos de enfrentar a otra cuestión: ¿Cómo es que efectivamente nuestras razones para actuar se ven reflejadas en la práctica en una acción por nuestra par-

\footnotetext{
${ }^{2}$ Entre las defensas del externismo de las razones se puede contar Scanlon, Th. M., What We Owe to Each Other, Belknap, Harvard, 1998; Parfit, Derek, «Reasons and Motivation», Supplement to the Proceedings of The Aristotelian Society, 71, 1997, 99-130.
} 
te? Dicho con otras palabras: ¡cuál es la relación entre nuestra acción y nuestra aceptación de una cierta razón para actuar? ¿Cuándo podemos decir realmente que asumimos una cierta razón para actuar, esto es, una posición moral? La respuesta que quepa dar a esta cuestión es la que determina que adoptemos una posición o bien internista o bien externista de la motivación moral. Comencemos por el internismo (IM) ${ }^{3}$. Este lo podemos caracterizar del modo que sigue:

(IM) Aceptar que tenemos una razón para actuar implica tener una motivación para actuar conforme a ella. Esto es, no asumimos realmente que tenemos una razón para obrar si no estamos realmente motivados para seguir. En otras palabras, el reconocimiento de nuestras razones para actuar no puede ser una tarea meramente epistemológica, sino que implica asimismo la participación una dimensión preferencial con respecto al estado de cosas del que tales razones se predican.

Dada esta definición, como cabe suponer, el externismo de la motivación moral será la posición contraria, esto es, $(\mathrm{EM})^{4}$ :

(EM) Aceptar que tenemos una razón para actuar no implica tener una motivación para actuar conforme a ella. Podemos aceptar que tenemos una cierta razón para actuar sin que, sin embargo, nos sintamos motivados a actuar conforme a esta.

Supongamos que tenemos la intención de obrar de un cierto modo, pese a que ello nos implica una serie de incomodidades muy notables. Imaginemos que, finalmente, cuando llega el momento de actuar de tal modo, las reticencias a actuar que nos provoca la perspectiva de tales incomodidades nos llevan a renunciar a actuar del modo en que teníamos pensado. ¿Cómo podemos interpretar lo que sucede en un caso así? Podemos hacerlo de dos modos.

\footnotetext{
${ }^{3}$ Una posición internista clásica sería la de Hare. Véase por ejemplo Essays in Ethical Theory, Oxford University Press, 1989.

${ }^{4}$ Entre los defensores del externismo de la motivación podemos encontrar a McNaughton, D., Moral Vision: An Introduction to Ethics, Blackwell, Oxford, 1988 y Dancy, J., Moral Reasons, Blackwell, Oxford, 1994, Practical Reality, Oxford University Press, Oxford, 2000, Ethics without Principles, Oxford University Press, Oxford, 2006.
} 
(a) Podemos decir que efectivamente nuestra intención era actuar, pero que la debilidad de nuestra voluntad nos ha impedido obrar como realmente queríamos. Si sostenemos que esto es lo que ocurre, seremos externistas de la motivación moral. Creeremos que hay una diferencia entre asumir una posición moral y obrar conforme a ella. Hay algo que media entre ambas instancias, que no es otra cosa que nuestra motivación. Así, distinguiremos un plano normativo, que será aquel en el que adoptemos nuestras posiciones morales, y uno motivacional, de carácter psicológico, que tendrá que ver con la forma en la que ponemos en práctica nuestras convicciones normativas.

(b) Por otra parte, podemos entender que lo que ocurre es que, si no hemos actuado conforme a la posición que en principio parecíamos haber adoptado, es que realmente nunca hemos asumido esta de veras. La perspectiva de nuestras incomodidades no es algo externo a nuestra consideración de nuestras razones para actuar, sino que entra en ellas. Nuestra motivación para obrar de un modo u otro forma así parte de nuestra consideración del modo en el que debemos obrar. De este modo, la motivación desempeña su papel no de manera externa a la adopción de una cierta posición moral, sino como un elemento central en esta. Así, no se distingue entre el plano normativo y el motivacional ${ }^{5}$.

Si aceptamos la primera descripción de lo que sucede seremos externistas de la motivación moral. Consideraremos que una vez que asumimos una determinada posición normativa, para que actuemos conforme a ella es necesario que entre en juego un factor externo a ella, esto es, una motivación psicológica. Si aceptamos, por el contrario, la segunda explicación, seremos internistas de la motivación, y entenderemos que esta juega su papel como parte de nuestra adopción de una u otra posición.

La distinción entre este problema y el de las razones para actuar es clara. Es por este motivo por el que cabe diferenciar entre dos posiciones susceptibles de ser denominadas con los términos «internismo» y «externismo»"

\footnotetext{
${ }^{5}$ Identificamos aquí lo moral con lo normativo, no con la parte de lo normativo que se refiere a nuestras relaciones hacia otros. Nuestras acciones autodirigidas formarían también parte de esta categoría.

${ }^{6} \mathrm{Ha}$ de resultar claro que la adopción de la segunda de las premisas que lo caracterizan no implica la de la primera. Es posible creer que podemos tener razones externas para actuar y entender, a
} 


\section{La filosofía moral abelardiana}

Una vez presentados los dos problemas teóricos centrales a los que nos enfrentamos podemos pasar a abordar la pregunta de cuál es su reflejo en la obra de Pedro Abelardo. Para tratar esta cuestión, comenzaremos por presentar las posiciones adoptadas por este en filosofía moral relevantes para la cuestión. El modo en el que abordaremos tal tarea será mediante el repaso de una serie de conceptos centrales en su pensamiento cuyo examen nos proporcionará una panorámica amplia del marco teórico en el que se encuadran.

Vamos a comenzar por el significado que Abelardo concede a los conceptos vicio y virtud. Estos, lejos de utilizarse en el sentido habitual, significan para Abelardo disposiciones que no dependen de la actividad humana, sino que son previos a ella. Dice Abelardo: «llamamos "costumbres" a los vicios y virtudes del alma que nos hacen propensos a obras malas o buenas»" . Así, el ser humano será, por naturaleza, vicioso y/o virtuoso, no siendo ya hábitos operativos buenos o malos como en Aristóteles.

Pasando ahora a relacionar estos conceptos con el de pecado podremos dibujar cuál es el fundamento del valor moral de la conducta humana para este autor. Para él, «vicio del alma no es lo mismo que pecado ni es el pecado lo mismo que la acción mala» ${ }^{8}$.

Acerquémonos ahora a la diferencia entre vicio, pecado y acción mala. El vicio es lo que nos hace proclives al mal. Constituye una tendencia, un impulso interior cuyo influjo experimentamos al margen de nuestra voluntad. Dice Abelardo: «Vicio es así aquello por lo cual nos volvemos propensos a pecar, esto es, nos inclinamos a consentir en lo que no se debe, haciendo algo o dejándolo de hacer»' Se trata así

\footnotetext{
la vez, que el reconocimiento de que tenemos una acción para actuar resulta inmediatamente motivador. No parece que sea inconsistente sostener estas dos ideas a la vez. Ahora bien, si, por el contrario, consideramos que todas nuestras razones para actuar han de ser de carácter interno, parece ya más difícil que podamos asumir que el aceptar que tenemos una acción no sea algo que deba motivar de forma inmediata, si bien puede seguir sosteniéndose que es así.

${ }^{7}$ Abelardo, P: Ética o conócete a ti mismo, Altaya, Barcelona, 1994, pág. 111.

${ }^{8}$ Op. Cit., pág. 114 .

${ }^{9}$ Op. Cit., pág. 116.
} 
de un fenómeno cuya evaluación cae fuera del ámbito normativo. El motivo es que constituye una inclinación natural inevitable. La existencia del vicio puede resultar negativa, pero no es en sí mismo algo que merezca la evaluación moral, al existir independientemente de lo que podamos hacer al respecto. Y toda prescripción que podamos hacer tendrá que asumir tal incapacidad por nuestra parte. Sin embargo, los agentes morales tienen dominio sobre estas inclinaciones, pudiendo decidir su actuación de modo consciente y libre. Y es aquí donde el ámbito normativo hace aparición. Según el agente acceda o no a guiarse por el vicio, así podremos decir que su acción es correcta o incorrecta. Se introduce así otro concepto central en la ética abelardiana, el de consentimiento. Según Abelardo, «consentimos verdaderamente en lo que no es lícito cuando de ningún modo nos retraemos de su realización y estamos interiormente dispuestos a llevarlo a cabo si se da la posibilidad $»^{10}$. Así, tiene lugar la acción moralmente incorrecta. O, dicho en otras palabras, conforme al esquema conceptual del autor, el pecado. La relación entre este y el consentimiento se ve claramente en las siguientes palabras de Abelardo: «Nada puede manchar el alma sino aquello que le es propio, es decir, el consentimiento, que es lo único que hemos denominado "pecado" y se sitúa en la voluntad que precede o sigue a la ejecución del acto»" ${ }^{11}$.

Entendemos así por qué puede decirse que la noción central del pensamiento abelardiano sea la de intención. Para Abelardo, «el mérito o la gloria no están en la obra misma sino en la intención del que obra» ${ }^{12}$ de tal modo que "cuando se habla de una buena intención y de una buena acción (esto es, de una acción que procede de una buena intención) sólo se designa una bondad, la de la intención»" ${ }^{13}$. Si acaso estos conceptos no han quedado suficientemente claros todavía es posible identificarlos con otro con el que seguramente estemos más familiarizados, esto es, la voluntad. Además, es nuevamente posible relacionar este concepto con la noción de pecado y virtud en palabras del autor. Dice así: «La voluntad de realizar una obra mala es pecado..., la voluntad de realizar una obra buena nos hace justos (de manera que así como la virtud consiste en la buena voluntad así el pecado consiste en la voluntad mala) ${ }^{14}$.

\footnotetext{
${ }^{10}$ Op. Cit., pp. 122-123.

${ }^{11}$ Op. Cit., pág. 130.

${ }^{12}$ Op. Cit., pág. 133.

${ }^{13}$ Op. Cit., pág. 157.

${ }^{14}$ Op. Cit., pp. 116-117.
} 
Asimismo, es necesario aclarar el término de acción mala, partiendo del hecho de que la acción no añade nada al valor moral, ya que el centro de este se sitúa en la intención. Así, la realización externa no tiene valor moral, residiendo este en la interioridad del sujeto, de tal modo que no hay acciones en sí mismas buenas o malas, sino acciones que proceden de una buena o mala voluntad.

Otro aspecto que puede resultar relevante para el estudio de la cuestión radica en la separación que realiza Abelardo entre la justicia humana, aquella que se ha de dar en el mundo, en las sociedades reales; y la justicia divina, en donde Dios se encargará de conservarla. El primer tipo estará basado en una ética de con reglas morales rígidas que permitan una vida en sociedad ordenada. Sin embargo, la justicia divina será la que contenga propiamente los juicios acerca de la intención del sujeto, avisando además el autor que los seres humanos por mucho que lo pretendan no están en disposición de tales juicios. ${ }^{15}$

De todas estas explicaciones podemos deducir que Abelardo, en contraposición con los moralistas de su época, no se preocupó de la casuística, sino de la raíz o fundamento de la moralidad (siendo esta una de las razones de la polémica levantada por su obra, como se puede ver en las constantes réplicas de su adversario Bernardo de Claraval).

Como podemos ver, la noción central del pensamiento abelardiano es la intención, que será juzgada por Dios — por motivos, tanto de hecho como de derecho: en la Tierra no se poseen los métodos necesarios, siendo además una tarea divina, que excede a los mortales_- Así, las acciones morales no tendrían peso, sino solamente las intenciones que mueven a realizar esa acción.

${ }^{15}$ Por otro lado, un aspecto interesante que es necesario señalar, es la existencia de personas con dificultades para realizar determinadas acciones, sea cual sea su intención. Existen trastornos que no permiten al individuo actuar como él quiere. Es verdad que es posible justificar esta deficiencia aludiendo a la excepcionalidad de estos casos, pero, con ello, se está dejando fuera un grupo no poco numeroso de individuos. Estos miembros de la sociedad no podrían en ningún caso ser juzgados de un modo negativo, ya que sus intenciones son buenas, pero tampoco de un modo positivo, debido a la falta de actuación posterior correspondiente a dichas intenciones. En este caso concreto, Abelardo opta por dejarlos fuera del análisis moral, pero ¿es esto lo correcto? 
Por último, podemos resaltar el carácter pragmático del autor en la medida en que establece dos justicias, a saber: la mundana y la divina. Con ello muestra que es consciente de los problemas derivados de la intención o, más bien, de un sistema basado en ella. Frente a esto plantea un modelo deontológico para la vida en la Tierra. No obstante, cabe preguntarse por la implantación de la ética de la intención en una sociedad utópica, donde los individuos estuviesen lo suficientemente desarrollados moralmente, fuesen virtuosos en este sentido, como para ser honrados y mostrar siempre su intención, y esta, en consecuencia, pudiese ser siempre captada por el resto. Cada individuo actuaría de un modo correcto, es decir, movido por una buena intención, comprendida por todos, pese a haber podido causar algún daño. Tal utopía, sin embargo, choca con la antropología abelardiana, conforme a la cual el ser humano es por naturaleza vicioso.

Llegados aquí, podríamos sostener que la posición abelardiana, dada la primacía que otorga al rol de la intención, se posiciona en coordenadas internistas. De hecho, podría plantearse esto en términos drásticos considerando que quizás desde un punto de vista como el de Abelardo, el hecho de que una persona dañe a otra con la intención de mejorar su vida no podría reprochársele, ya que su intención era buena. Es verdad que la juzgamos como egoísta, pero el hecho es que su intención era un bien. Lo mismo ocurre en el caso de dañar a otra persona por el mero hecho de verla sufrir. La intención podría ser buena, ya que lo que se busca es el beneficio o satisfacción propia más que el daño ajeno. Es por ello que cualquier acción derivada de una intención, así como todas y cada una de las conductas del sujeto serían loables.

Más aun, a la luz de esto podríamos incluso dudar de si el planteamiento abelardiano sería no ya internista, sino incluso subjetivista ${ }^{16}$. El subjetivismo es la posición que afirma que las propiedades morales son de carácter subjetivo. Podríamos plantearnos si la teoría ética abelardiana consistiría en una versión de esta posición. Dado que «solamente hay pecado contra consciencia» ${ }^{17}$, podríamos pensar que efectivamente sólo en la medida en que se asuma una cierta posición puede efectivamente ser juzgada nuestra práctica moral. De este modo, lo que

\footnotetext{
${ }^{16} \mathrm{Si}$ bien todas las posiciones externistas han de ser necesariamente objetivistas, el internismo es compatible con planteamientos tanto subjetivistas como objetivistas.

${ }^{17}$ Abelardo, P. Conécete a ti mismo.
} 
parece que sucede es que nuestro criterio normativo depende de nuestra aceptación de unas razones u otras para obrar. Se podría incluso considerar si Pedro Abelardo es un subjetivista que defiende la intersubjetividad, en cuanto que los valores morales son defendidos por la comunidad en su totalidad -la religiosa-, pero desde cada individuo concreto, no existiendo en sí. Más aun, quizás podríamos recordar en este punto el hecho de que el autor critica el realismo en lo que toca al problema de los universales, trasladado al ámbito lógico-lingüístico para acentuar el valor de lo individual y concreto (estableciéndose con ello la base de la epistemología empirista no dogmática que luego será desarrollada por Ockham, Locke y Hume). Cabría pensar, en este sentido, que sus posiciones en metaética y psicología moral deberían ir en línea con esto; a fin de cuentas, esta parecería, como mínimo, la posición más parsimoniosa. De ser así, podríamos sostener que la postura obvia a sostener por parte de Abelardo sería la oposición al realismo moral, lo cual, en lo que atañe a nuestras razones para actuar, nos llevaría a abrazar una posición externista. Sin embargo, tal paralelismo entre los planos ontológico y lógico y el moral no se sigue de forma necesaria.

Así, a favor del planteamiento externista de Abelardo puede apuntarse, simplemente, su planteamiento cristiano. Esto no viene dado meramente por la creencia en una realidad metafísica más allá de nuestra percepción, claro está, sino por el contenido moral que el cristianismo defiende que tal realidad tendría. Conforme a este punto de vista existen una serie de valores establecidos que han de seguirse. Estos no son fijados por la comunidad religiosa, sino que radican en propiedades morales de carácter sobrenatural. Se ha de actuar del modo que el credo asumido dicta y existen razones externas al individuo para hacerlo.

Por supuesto, puede indicarse aquí que, desde la ignorancia, podría hacerse lo que en principio no estaría prescrito por la divinidad, y que ello, al ser la intención lo considerado, no resultaría condenable. En realidad, no explicita con claridad su posición Abelardo, si bien hay razones para considerar que esta implicaría la salvación de quienes no reconociesen o desconociesen los dogmas de la fe cristiana. Ahora bien, pero si esto es así, ¿no habría motivos para dudar de que la posición abelardiana fuese externista?

En realidad, no es difícil comprobar que no es así. Quienes se comportan de forma contraria no es que tengan una motivación propia para actuar epistemológicamente tan válida como cualquier otra. Sino que por el contrario, se encuentran 
equivocados. Existen unos hechos sobre aquello que hemos de hacer, y hay quienes se equivocan al considerarlos. Lo único que sucede es que, en la interpretación más indulgente del planteamiento abelardiano, tal equivocación sería perdonada.

Finalmente, hay otro punto en el que esto se comprueba. Este consiste en la aceptación abelardiana de que exista un pecado que no pueda ser nunca perdonado por Dios. Así, mientras que mediante la penitencia y el arrepentimiento la gran mayoría de los pecados pueden ser lavados, hay uno en concreto con el que no ocurre así: la blasfemia. Abelardo acepta que blasfemar contra el Espíritu Santo sería un pecado tan horrendo que quien sea capaz de cometerlo ya no se arrepentirá. Pero esta posición es evidentemente implausible. Parece a todas luces que estamos aquí ante un argumento ad hoc para intentar evitar las consecuencias más radicales (desde la perspectiva abelardiana) de la aceptación del planteamiento centrado en la intención. Si esto es realmente así, tenemos una nueva razón para considerar que Abelardo no puede asumir realmente un enfoque internista.

\section{El lugar de la motivación moral para Abelardo}

En principio podría pensarse que tenemos motivos para considerar a Abelardo un externista de la motivación moral. Ello radicaría en la clara distinción trazada por este entre el ámbito de nuestros impulsos naturales y el de nuestras decisiones acerca de estos. Podríamos considerar que esta separación de ámbitos sería paralela a la que se daría, en el debate en torno a la motivación moral, entre el ámbito meramente psicológico y el normativo.

Sin embargo hay motivos para dudar de esta afirmación. Consideremos el siguiente caso: supongamos que asumimos que debemos llevar a cabo una cierta acción (por ejemplo, para ayudar a alguien), imaginemos que a la hora de llevar tal acción a la práctica nuestra voluntad nos falla y somos incapaces de hacerlo. ¿Qué evaluación cabrá hacer con respecto al planteamiento normativo de Abelardo de una omisión como esta en nuestra acción? Parece que no hemos actuado debido a que tenemos un impulso vicioso que nos lleva a ello (la vagancia, por ejemplo). Ante esto podemos, por una parte, decir que somos inocentes y no hemos incurrido en una acción incorrecta. El motivo para tal afirmación pasaría por el hecho de que, como hemos visto, para Abelardo el lugar de la práctica moral no es la intención. Así, el motivo por el que la adopción de una posición moral no se habría plasmado en una 
acción real radicaría en que sería algo externo a esta, en una circunstancia no moral. Ahora bien, la suposición de lo que nos llevaría a la inacción, sería una instancia no moral que resulta seguramente extraña. Podemos creer que Abelardo no la aceptaría. En el ejemplo, Abelardo concluiría que deberíamos ser juzgados negativamente. Lo que nos llevó a la inacción, conforme a este, parece que no puede ser meramente nuestro impulso, sino nuestra cesión ante este. De tal modo, la voluntad entra en juego, y pasamos a poder ser juzgados moralmente. Conforme a esto, podríamos decir que el motivo básico para actuar de forma incorrecta sería, más bien, la existencia de razones de otro tipo que constituirían un obstáculo a la hora de guiarnos en exclusiva por las razones relativas. Si esto es así, pasamos a tener que concluir que Abelardo no podría asumir una posición externista de la motivación moral.

El examen de una posición como la abelardiana nos proporciona un argumento a favor del internismo en el siguiente sentido: en la medida en la que rechacemos el realismo moral y consideremos que nuestras prescripciones morales no vienen de un plano independiente de nuestra voluntad. Si afirmamos esto, es lógico que no tengamos reparo alguno en incluir nuestras inclinaciones en la determinación de nuestras posiciones morales.

\section{Conclusiones}

Para finalizar articulando ambos planteamientos, podemos señalar que la problemática metaética a la que nos hemos referido a lo largo de este artículo es propia de los debates contemporáneos sobre la cuestión, y desde luego no fue reconocida como tal por Abelardo, que no disponía realmente del instrumental teórico para llegar a formularla. Esto podría hacer parecer una investigación como la desarrollada aquí como incongruente, fuera de lugar o, en el mejor de los casos, poco informativa, o meramente una yuxtaposición de ideas. Sin embargo, entendemos que no es así. No sólo lanzamos hipótesis, sino que consideramos que el fenómeno al que se enfrentaba el análisis de Abelardo es el mismo que tiene como objeto la reflexión llevada a cabo hoy en día por los filósofos morales de la actualidad. Tanto en el siglo XII como en el XXI existen agentes que consideran que sus acciones no son aleatorias, sino que se deben a ciertas razones. El modo en el que se comprenda cuál es la naturaleza de tales razones, y de la conexión con su asunción y nuestra motivación puede variar. Y sobre todo puede hacerlo su presentación, y la clase de conceptos de base que se adoptan para su abordaje. Pero el problema de fondo, en la medida en 
la que lo que se esté examinando sea la fundamentación de la filosofía moral, es este mismo. Si las preguntas que hemos planteado a Pedro Abelardo no tuviesen sentido, ello significaría que este no habría estado realmente tratando esta cuestión. Sin embargo, el hecho es que sí tienen sentido. Y lo tienen porque en el pensamiento abelardiano hay una concepción de estos problemas. Incluso aunque Abelardo no los aborde explícitamente, sostiene una posición al respecto. De este modo, es posible extraer una reflexión y conclusión a partir del interrogatorio al que hemos sometido a su obra.

Esta aclaración cobra mucho más sentido cuando constatamos que efectivamente cabe derivar conclusiones sustantivas de interés del examen que hemos llevado a cabo aquí. Hemos comenzado nuestro examen de la cuestión definiendo el internismo y el externismo de manera general, para a continuación distinguir dos premisas en la formulación de cada una de estas posiciones, que llevan a diferenciar dos problemas distintos, relativos a nuestras razones para actuar y al carácter de la motivación moral. Una vez trazado este marco conceptual pudimos concluir que Abelardo sostiene una posición externista (con lo que damos respuesta al título del artículo) tanto en lo que toca a las razones para actuar como en lo que atañe a la motivación moral. Ello nos proporciona una base interesante para ponderar la relevancia que puede tener la alusión a la intención como concepto central en filosofía moral. Mediante el análisis aquí desarrollado se ha mostrado que la relevancia en filosofía moral resulta menos determinante de lo que podría parecer a primera vista. Como hemos visto a la luz del análisis de la propia obra de Abelardo, el interés normativo de una concepción de este tipo puede ser muy reducido, al no determinar una guía de acción concreta. Ante esto, podríamos considerar que la relevancia de esta dimensión radica en su aportación de cara a comprender la naturaleza del fenómeno moral. Vimos, sin embargo, que también su utilidad a tal respecto será más moderada de lo que podría parecer. Si bien a la hora de asignar responsabilidades esta categoría resulte central, en lo que toca a la comprensión de la ontología de la moral es una categoría que en sí misma resulta insuficiente.

\section{Bibliografía}

Abelardo, P. (1994). Ética o conócete a ti mismo, Barcelona, Altaya.

Abelardo, P. (1988). Diálogo entre un Filósofo, un Judio y un Cristiano, Zaragoza, Yalde. 
ChÉnu, M.D. (1957). La Theologie au douziéme siecle, París, Vrin.

DANCY, J. (1994). Moral Reasons, Oxford, Blackwell.

- (2000). Practical Reality, Oxford, Oxford University Press.

- (2006). Ethics without Principles, Oxford, Oxford University Press.

Hare, R. (1989). Essays in Ethical Theory, Oxford, Oxford University Press.

HeIl, J. \& A. Mele (1995). Mental causation, New York, Oxford University Press.

Huizinga, J. (1960). Hombres e Ideas. Buenos Aires, Compañía Fabril Editora.

Harman, G. (1977). The Nature of Morality, Oxford, Oxford University Press.

- (1997). «Reasons and Motivation», Supplement to the Proceedings of The Aristotelian Society, 71, 99-130.

Harman, G \& J. ThOMSON, J. (1996). Moral Relativism and Moral Objectivity, Cambridge, Blackwell.

KorsgaARD, CH. M. (2000). Las fuentes de la normatividad, México, UNAM.

Mcnaughton, D. (1988). Moral Vision: An Introduction to Ethics, Oxford, Blackwell.

PARFIT, D. (1997). "Reasons and Motivation», Supplement to the Proceedings of The Aristotelian Society, 71, 99-130.

Persson, I. (2005). The Retreat of Reason: A Dilemma in the Philosophy of Life, Oxford, Oxford University Press.

PRINZ, J.J. (2007). The emotional construction of morals, Oxford, Oxford University Press.

SCANlON, Th. M. (1998). What We Owe to Each Other, Harvard, Belknap.

Singer, P. (Ed.) (1995). Compendio de ética, Madrid, Alianza.

SMith, M. (1996). «The Humean Theory of Motivation», Mind, 96, 1987, 36-61.

- (1996): The Moral Problem, Oxford, Blackwell.

Williams, B. A. O. (1978). Morality: An Introduction to Ethics, Cambridge, Cambridge University Press.

- (1981). «Internal and External Reasons», en Moral Luck: Philosophical Papers 19731980, Cambridge, CUP, 101-113.

Recibido: 1/03/2011

Aceptado: 19/10/2011 
